\title{
Staphylococcal septicaemia, endocarditis, and osteomyelitis in dialysis and renal transplant patients
}

\author{
ANTHONY Nicholls \\ M.B., M.R.C.P.
}

NEIL EDWARD
M.B., F.R.C.P.E.

\author{
Graeme R. D. CAtTo \\ M.D., M.R.C.P.
}

University Department of Medicine and Medical Renal Unit, Royal Infirmary, Aberdeen

\begin{abstract}
Summary
Septicaemia is a common and potentially lethal hazard of haemodialysis and renal transplantation; it is usually caused by Staphylococcus pyogenes. In 6 patients with $S$. pyogenes septicaemia, fatal endocarditis and spinal osteomyelitis have each occurred once, and 3 patients have had recurrent episodes of septicaemia. The managment of septicaemia in these patients must include a search for metastatic infection, and prolonged therapy with 2 antistaphylococcal agents is necessary to ensure eradication of infection. Access site infection in dialysis patients must be treated vigorously, and recognized as potentially hazardous by patients. The risk of sepsis in dialysis and transplant patients cannot be excluded, but devastating consequences may be avoided by simple measures.
\end{abstract}

\section{Introduction}

Immune competence is characteristically depressed in uraemia (Wilson, Kirkpatrick and Talmage, 1965; Lawrence, 1965; Dobbelstein, 1976), with a correspondingly enhanced susceptibility to infection (Montgomerie, Kalmanson and Guze, 1968). Regular dialysis might be expected to correct this depression of the immune response (Newberry and Sanford, 1971) but this has not been demonstrated (Gombos, Jefferson and Bhat, 1975) and frequent infections are common in dialysis patients. Following renal transplantation, the use of immunosuppressive drugs is accompanied by a constant threat of serious infection which is the leading cause of death in transplant patients, and the second most common cause of death in patients on dialysis (Wing et al., 1978).

In addition to conventional infections of the skin, chest and urinary tract, dialysis patients face the special hazard of access site infection. Apparently trivial sepsis at a shunt site or overlying a subcutaneous fistula may herald more serious complications including septicaemia (Ralston et al., 1971;
Dobkin, Miller and Steigbigel, 1978), septic pulmonary emboli (Levi, Robson and Rosenfeld, 1970), infective endocarditis (Cross and Steigbigel, 1976; Lavelle and Dentino, 1978) and osteomyelitis (Leonard et al., 1973a). Staphylococcus pyogenes is the usual causal organism in such cases, although Gram-negative bacilli are also encountered (Dobkin et al., 1978). Total eradication of micro-organisms may prove difficult if an infective nidus exists in bone or on a heart valve; when associated with endarteritis of the fistula itself, the fistula may nee $\$$ to be removed if disseminated disease is to be managed successfully (Cross and Steigbigel, 1967) Recurrent episodes of septicaemia can occur in dialysis patients (Levi et al., 1970); they may harbour quiescent foci of infection which can be reactivated by immunosuppressive therapy following renal transplantation.

This paper deals with $S$. pyogenes septicaemia in a dialysis and transplant unit. Over a 3-year period the authors have seen 11 septicaemic episodes in 6 patients, 8 of these episodes having occurred during 1978.

\section{Patients and methods}

At the end of the 3-year period 1976-1978, 20 patients were on home dialysis, 16 on hospital dialysis and 21 had functioning renal transplants. Twenty-five patients dialysed using a standard radial or ulnar Cimino-Brescia type fistula; 7 patients used implanted woven polytetrafluoroethylene (Gore-tex) grafts for access; one had a bovine xenograft; and 2 used Scribner shunts in the leg. Blood cultures were only considered positive if both of a simultaneous pair of culture bottles grew a typical pathogen in an appropriate clinical setting. There were 11 cases of $S$. pyogenes septicaemia ( 2 episodes of septicaemia due to Escherichia coli which occurred in renal transplant recipients are not reported here). 


\section{Case reports}

\section{Case 1}

A 34-year-old man with Alport's syndrome developed a febrile illness in October 1976, one month after being transferred to home dialysis; his most prominent uraemic symptom had been intractable pruritus. Several blood cultures grew $S$. pyogenes: gentamicin was given for 4 days and oral flucloxacillin for 2 weeks. Two months later an identical bacteraemic illness was treated with 2 weeks of cloxacillin at first parenterally and later orally. Three months later he complained of thoracic backache; initially the X-rays were considered as showing only features of renal osteodystrophy (Fig. 1), but his back was explored as osteomyelitis could not be excluded. Pus aspirated from a thoracic vertebra grew $S$. pyogenes on culture. Cloxacillin $2 \mathrm{~g}$ daily for 8 weeks settled his backache. In April 1978 his right knee became painful and swollen; no fluid could be aspirated, and blood cultures were sterile, but flucloxacillin was given for 2 weeks. One month later he had a further febrile illness with backache, right knee pain and right shoulder pain. $S$. pyogenes was again isolated from his blood, and he was treated with gentamicin and cloxacillin for 4 weeks. Renal transplantation was performed a few weeks later, and after 18 months he remains well. $S$. pyogenes has not been isolated from his skin or nose.

Comment. This patient had severe pruritus during regular dialysis therapy, and developed septicaemia from skin sepsis. Inadequate early therapy of his septicaemias allowed metastatic spread to bone. His uneventful course following transplantation probably reflects relief of pruritus, and there is now no skin carriage of $S$. pyogenes.

\section{Case 2}

A 56-year-old man with chronic pyelonephritis developed an itchy infection lesion on his left leg. He then developed a florid septicaemic illness, with acute-on-chronic renal failure, and $S$. pyogenes in blood cultures. He was treated with cloxacillın and erythromycin for 5 weeks. He was also dialysed, first through a Scribner shunt in the right leg, and then via a standard radiocephalic fistula as there was no return of renal function. Nine months later, in April 1978, he felt unwell and had a low grade

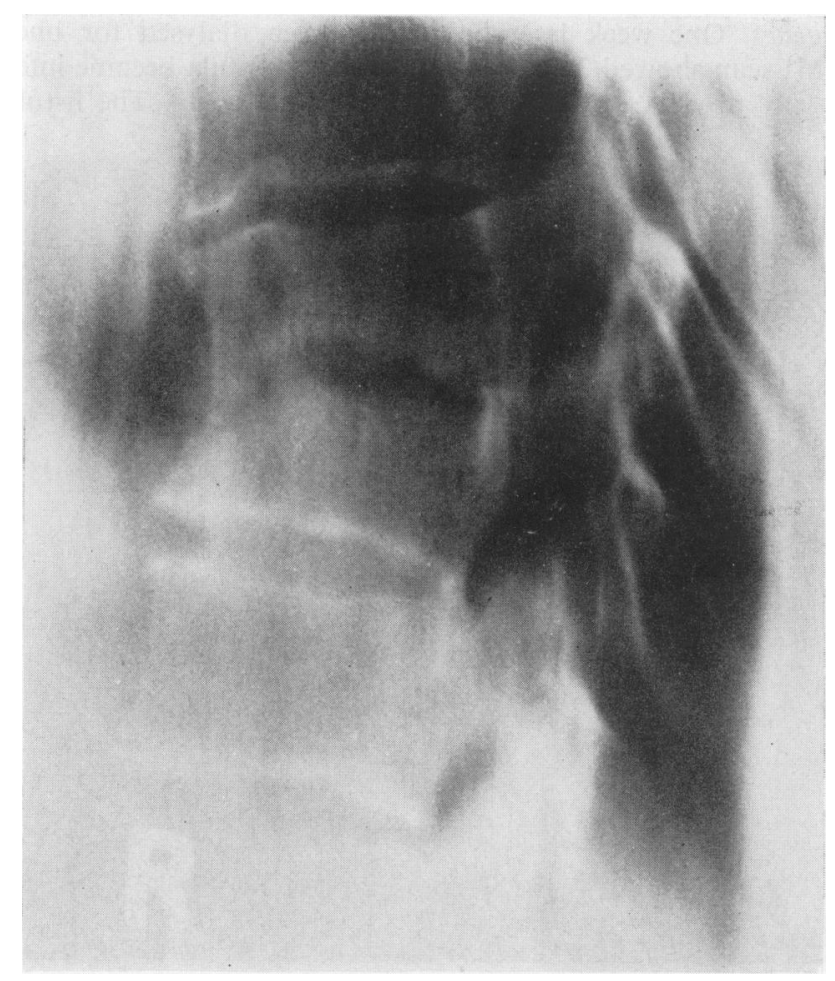

FIG. 1. Case 1: Lateral tomogram of thoracic spine showing destruction of the lower border of T8 and the upper border of T9 with loss of the disc space. 
fever. His right shoulder was painful, and he had a right olecranon bursitis. $S$. pyogenes was grown on repeated blood cultures, and he received cloxacillin for 3 weeks. In August 1978 he developed fever, malaise and pericarditis. Echocardiography showed a small pericardial effusion, but normal heart valves. $S$. pyogenes was grown in his blood, and he was treated with cloxacillin and erythromycin for 3 months. He has remained well for $>$ one year, and is not a nasal or skin carrier of $S$. pyogenes.

Comment. His original septicaemia was probably under-treated; subsequent episodes might have been averted with more prolonged initial therapy. Since receiving antibiotics for 3 months there have been no further problems.

\section{Case 3}

In May, 1978, a 40-year-old male with obstructive uropathy damaged his fistula with a needle, but dialysed successfully. Three days later he had a high fever, breathlessness, confusion, extensive purpura on his trunk, splinter haemorrhages and a left pleural effusion; the heart was clinically normal. $\mathrm{He}$ was given i.v. cloxacillin and gentamicin on admission to hospital. Swabs from a septic lesion overlying his fistula, pleural aspirate, and blood cultures all grew $S$. pyogenes. One week later he remained confused; an EMI scan showed 2 areas of cerebral infarction, but no abscess. There was still no clinical cardiac abnormality. A further specimen of pleural fluid grew an unidentified Staphylococcus sensitive to cloxacillin and gentamicin. Three weeks after admission he had signs of aortic incompetence, confirmed by echocardiogram, which also showed valvular vegetations (Fig. 2). Blood cultures were now negative, but his condition deteriorated steadily and cardiac surgery was considered too hazardous. In midJune, a new, louder systolic murmur appeared, and he rapidly developed progressive and terminal cardiac failure. Post-mortem revealed acute bacterial endocarditis affecting aortic, mitral and tricuspid valves, the foci of infection being linked by a myocardial abscess (Fig. 3). The clotted fistula was sterile, but a scanty growth of $S$. pyogenes was isolated from the heart.

Comment. The source of infection was the fistula, which had become infected after faulty needle insertion. Emergency heart surgery might have saved this patient, as he died despite appropriate antibiotic therapy.

\section{Case 4}

A 37-year-old male with obstructive uropathy had been dialysed for one year when, in Marcl 1977 , his fistula became infected around a clot-linee pseudoaneurysm. The fistula was excised, and ora

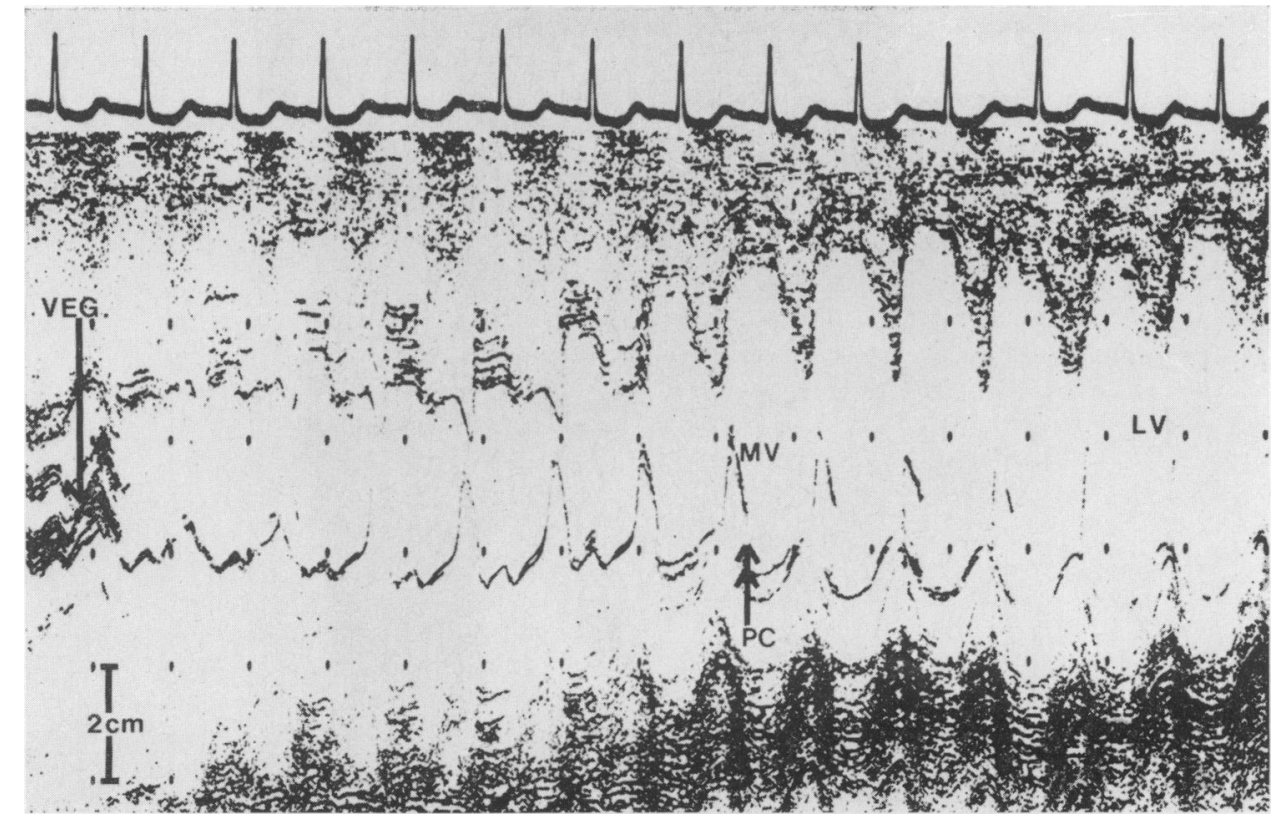

FIG. 2. Case 3: Echocardiogram. Aorto-mitral sweep showing aortic valve vegetations (VEG, single arrow), acute left ventricular dilation (LV) due to aortic regurgitation, and premature closure (PC, double arrow) of the mitral valve (MV). 


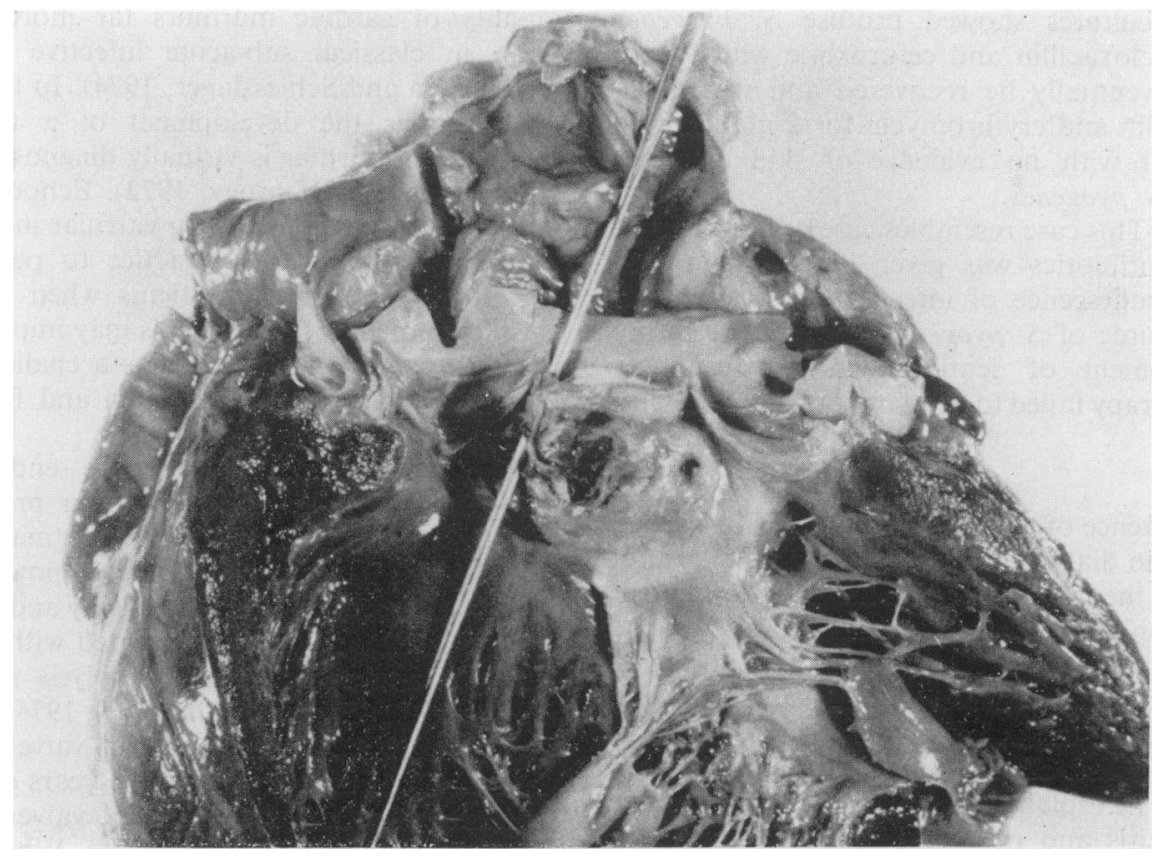

Fig. 3. Case 3: Post-mortem. The aortic root and left ventricle have been opened to show a large friable haemorrhagic vegetation on the ventricular aspect of the non-coronary cusp of the aortic valve. The probe lies through one of 2 one-cm perforations in the cusp.

flucloxacillin was given for 10 days. Renal transplantation was successfully performed in May 1978, but 5 months later, whilst on prednisolone $15 \mathrm{mg} /$ day and azathioprine $100 \mathrm{mg} /$ day, the patient developed malaise, fever and chills. $S$. pyogenes was isolated from blood, urine and a throat swab, and gentamicin and cloxacillin were given for 2 weeks. Two months later, $S$. pyogenes bacteraemia recurred, and a 2-month course of cloxacillin was given. He remains well, and nose, throat and skin swabs have not grown $S$. pyogenes on culture since the second illness.

Camment. The second septicaemia probably resulted from inadequate treatment of the previous episode. In the presence of immunosuppressive therapy, antibiotic treatment should be administered for longer than in other patients. His previously infected fistula was probably unrelated to the infections following transplantation.

\section{Case 5}

A 53-year-old man with a history of proteinuria and hypertension commenced haemodialysis in January 1978 via a shunt in the left leg. In September 1978 the shunt was removed because of local infection and he received flucloxacillin for 2 weeks. In November 1978 he presented with chest pain, malaise, fever, pericarditis and splenomegaly. A small pustule overlying his fistula had been present for 3 days, unreported by the patient. Pus from this lesion and blood cultures grew $S$. pyogenes sensitive to cloxacillin, which was given for 8 weeks following initial i.v. therapy. Echocardiography showed a small pericardial effusion but no valvular dysfunction. The fistula clotted a few days after admission, and dialysis is now effected via a right leg shunt.

Comment. The importance of sepsis overlying a fistula must be understood by patients: prompt therapy is essential if septicaemia is to be avoided. The authors have adopted a policy of prolonged antibiotic therapy for septicaemia in the light of their experience with the preceding patients.

\section{Case 6}

A 48-year-old man with renal failure of undetermined cause received a renal transplant in December 1977 following 10 years' haemodialysis. Postoperative complications included gastrointestinal haemorrhage and cytomegalovirus infection, but the patient recovered and remained well on azathioprine $50 \mathrm{mg} /$ day and prednisolone $17.5 \mathrm{mg} /$ day during 1978. In October and November he developed several boils on his trunk and neck which were treated by drainage and antibiotics. In December 1978 he became unwell for 3 days with malaise, lethargy, rigors and fever. Throat swabs 
and blood cultures showed profuse $S$. pyogenes sensitive to cloxacillin and cefuroxime which were given i.v. Eventually he recovered and was given oral cloxacillin and erythromycin for 2 months. He remains well with no evidence of skin or nasal carriage of $S$. pyogenes.

Comment. This case resembles case 4; a prolonged course of antibiotics was given in an attempt to avoid a recrudescence of infection. In contrast to case 4 , a source of $S$. pyogenes was evident before the development of septicaemia, yet seemingly adequate therapy failed to avert major complications.

\section{Discussion}

The occurrence of these 11 episodes of $S$. pyogenes septicaemia in dialysis and transplant patients is not exceptional; the incidence of septicaemia per patient per year of only $7 \%$ for the authors' dialysis patients compares favourably with that of $15 \%$ reported in a much larger series in New York (Dobkin et al., 1978). However, the much higher incidence during 1978 , the problem of recurrent septicaemia in both dialysis and transplant patients, and the occurrence of endocarditis and osteomyelitis has provoked a further examination of these issues.

\section{Endocarditis}

In the early days of chronic haemodialysis when Scribner shunts were the sole means of acess to the circulation, infective endocarditis was not described despite recurrent local sepsis. However, in the original account of the surgically-created subcutaneous arteriovenous fistula (Brescia et al., 1966), one of the 13 patients described died of infective endocarditis, and in 1969 Goodman et al. described 2 further cases of bacterial endocarditis in patients using fistulae for dialysis. Also in 1969, Kurtz described the first case of endocarditis associated with a shunt: the patient died.

Since these early reports there have been several other accounts of endocarditis in dialysis patients and, in a review article (Cross and Steigbigel, 1976), 35 episodes were collected in 34 patients. The outcome was stated in 29 patients, of whom 16 died. Twelve episodes of endocarditis were associated with subcutaneous fistulae, 20 with external shunts, and 3 occurred in patients with both types of access device. There is thus little support for the opinion that disseminated infection is a greater hazard from fistulae than from shunts (Ralston et al., 1971).

The recognition of endocarditis in dialysis patients can be difficult. However, one is likely to be dealing with acute rather than sub-acute or chronic disease: consequently the highly destructive vascular lesions will cause striking changes in the intensity and quality of cardiac murmurs far more commonly than in classical sub-acute infective endocarditis (Weinstein and Schlessinger, 1974). In the setting of acute sepsis the development of a new organic $\overrightarrow{\overline{\vec{S}}}$ regurgitant murmur is virtually diagnostic of infect- $\overrightarrow{0}$ ive endocarditis (Dorney, 1972). Echocardiography $\frac{\bar{\sigma}}{\sigma}$ is valuable in demonstrating valvular incompetence; $\frac{\overline{\bar{s}}}{\bar{a}}$ it is now the authors' practice to perform echo- $\frac{\widehat{ }}{\alpha}$ cardiograms on septic patients when there is any suspicion of endocarditis. This may improve diagno- कs sis of a condition that remains a challenge even in $\vec{\circ}$ non-uraemic patients (Weinstein and Rubin, 1973; $\overrightarrow{\vec{\omega}}$ Wann et al., 1976).

The management of infective endocarditis in $\bar{D}$ dialysis patients is no different in principle from 3 that in non-uraemic subjects. The major criterion ir for valve replacement is the development of severe cardiac failure (Hancock, Shumway and Remington, of 1971); cardiac failure is associated with $100 \%$ mor- os tality in non-operated haemodialysis patients with $\stackrel{\hat{N}}{\mathrm{~N}}$ endocarditis (Cross and Steigbigel, 1976; Lavelle and 음 Dentino, 1978). Experience with valve replacement is limited but the survival at 1-3 years of 13 dialysis $\&$ patients who have had infected valves replaced is $\frac{\mathbb{O}}{\mathbb{D}}$ $61 \%$ (Lavelle and Dentino, 1978). Whether a more aggressive approach with early surgical intervention is justified is not clear; but in a non-dialysis popub lation mortality in high risk patients with up $\vec{\bullet}$ controlled infection was reduced from $90 \%$ t. $17 \%$ when a policy of early surgery was adopte (Boyd et al., 1977).

\section{Osteomyelitis}

The second major issue concerns osteomyelitis. From the literature this is apparently an extremely unusual problem, having been reported from only one centre (Leonard et al., 1973a). The same authors record separately 4 other patients with osteomyelitis who had co-existing bacterial endocarditis (Leonard, Raij and Shapiro, 1973b; Leonard et al., 1973c). In these cases, eradication of the infecting organisms was a problem, and 2 patients died. The present authors agree with Leonard et al. that differentiation of osteomyelitis from renal osteodystrophy may be difficult, but in the present case the dilemma was resolved by exploration of the spine and the finding of pus. It is therefore advocated that any suspicious bony lesion which in the clinical setting might be due to osteomyelitis should be $\tilde{\sigma}$ explored early. In retrospect, in their case, the dura- N tion of antibiotic for previous episodes of septicae- N mia was too brief, and allowed haematogenous spread of infection to bone. Whether treatment of the osteomyelitis for longer than 8 weeks would have prevented a subsequent septicaemia seems doubtful, but more prolonged therapy might be given in future cases. 


\section{Management of infection}

The proper management of access site infections and septicaemia in dialysis and transplant patients is not yet clear. In particular, the optimum duration of therapy and choice of antibiotic have not been discussed in detail.

Provided that in vitro sensitivities are used as a guide, there is no firm evidence that the choice of antibiotic is crucial. For $S$. pyogenes infections in dialysis patients a wide variety of agents have been used, including penicillin, oxacillin, cloxacillin, gentamicin, vancomycin, chloramphenicol, cephalothin and fusidic acid (Leonard et al., 1973b; Rao et al., 1972; Barcenas et al., 1976) and no single agent has proved outstandingly successful. Similar conclusions have been drawn from studies of $S$. pyogenes septicaemia in a non-dialysis and transplant population (Wise, 1973; Nolan and Beaty, 1976). Thus, despite the apparent efficacy of potent antistaphylococcal antibiotics, treatment failures still occur. On an empirical basis, therefore, the present authors now favour the concurrent use of 2 antistaphylococcal agents: they use gentamicin or a cephalosporin with cloxacillin or penicillin. The limited evidence available suggests that such combination therapy may be effective (Andriole, 1979).

The optimum duration of antibiotic therapy for staphylococcal septicaemia in dialysis and transplant patients is not known. Many reports in the literature detail the antibiotic used, but not the duration of therapy (Dobkin et al., 1978; Levi et al., 1970; Sherrard, 1970). S. pyogenes septicaemia is notorious for the frequency with which endocarditis occurs; in $50 \%$ of cases normal heart valves are involved (Weinstein and Rubin, 1973) and the possibility of endocarditis exists in all patients with proved $S$. pyogenes septicaemia (Gregoratos and Karliner, 1979). The dictum of Lerner and Weinstein (1966) that $S$. pyogenes septicaemia should always be regarded as involving the heart, and treated accordingly, is widely quoted; 2 reviews recommend 3 to 6 weeks of i.v. antibiotic therapy in all cases, although none of the patients in these reports was a transplant recipient or on dialysis (Nolan and Beaty, 1976; Wise, 1973). Prolonged daily i.v. therapy in dialysis and transplant patients is often difficult owing to lack of veins. An attempt (unsuccessful) has been made to overcome this problem by using vancomycin (Barcenas et al., 1976).

Renal transplant recipients are most at risk from infection in the early weeks when immunosuppressive drugs are used in higher doses, but the authors' 2 cases of $S$. pyogenes septicaemia occurred some months after transplantation when the dose of immunosuppressants was modest.

It seems clear, therefore, that in all patients with $S$. pyogenes septicaemia, whether in the normal population, on dialysis or following transplantation, total eradication of infection may prove difficult, reflecting the extreme virulence of the organism. The authors' experience indicates that in dialysis and transplant patients, 2-4 weeks' therapy is insufficient to ensure cure. In both groups of patients this is likely to be due, at least in part, to impaired immunity even though high blood and tissue levels of antibiotics are easily achieved in anuric patients. The authors therefore favour 8 weeks' antibiotic therapy for $S$. pyogenes septicaemia in their dialysis and transplant patients, although formal validation of this approach is lacking.

Prophylactic oral antibiotic therapy has been used to reduce access site infections and their complications, but uncontrolled studies suggest that it is ineffective (Dathan et al., 1970; Martin et al., 1967). Prophylactic parenteral antistaphylococcal therapy reduces overt staphylococcal sepsis but predisposes to Gram-negative infections (Morris and Bilinsky, 1971). The epidemiology of access site infections and their complications has demonstrated that staphylococcal carriage and endogenous infection is the most likely explanation for repeated sepsis (Dobkin et al., 1978; Levi et al., 1970; Martin et al., 1967; McIntosh, Petrie and MacLeod, 1969; Morris and Bilinsky, 1971; Rebel et al., 1975), and most episodes of septicaemia in dialysis patients follow shunt or fistula infection. Although eradication of nasal carriage of staphylococci by topical antibiotics does not reduce the incidence of access-site infection (Martin et al., 1967; Morris and Bilinsky, 1971), elimination of skin carriage of staphylococci by hexachlorophane washing reduces the infection rate (Sherrard, 1970). During cannulation of a fistula, it is the occasional difficult needling associated with repeated attempts to enter the vein and consequent bruising that is most likely to predispose to local infection and subsequent septicaemia. In these circumstances, the procedure often becomes non-sterile (see case 3 ).

When an infected access-site gives rise to septicaemia or endocarditis, its removal may be necessary in order to control infection (Cross and Steigbigel, 1976). However, in view of the need to preserve vascular access for haemodialysis, this approach is undesirable-it has not been the authors' practice, nor that of other workers (Levi et al., 1970; Ralston et al., 1971; Sherrard, 1970).

The risk of infection will always be present in dialysis and transplant patients, irrespective of the precautions taken. However, simple measures including prompt and vigorous treatment of minor skin sepsis, prolonged antibiotic therapy with 2 drugs in cases of septicaemia, and a careful search for potentially lethal complications such as endo- 
carditis and osteomyelitis may be expected to reduce the morbidity and mortality from infection in these patients.

\section{Acknowledgements}

We would like to thank the staff of the Bacteriology Department for their help in the management of these cases; Dr J. Weir of the Radiology Department for the echocardiogram; Dr L. S. Jayasinghe, formerly of the Radiology Department, for the X-ray; Dr J. L. McPhie of the Pathology Department for the post-mortem photograph; and the secretaries of the Medicine Department for typing the manuscript.

\section{References}

ANDRIOLE, V.T. (1979) Staphylococci and combination therapy. Archives of Internal Medicine, 139, 1090.

Barcenas, C.G., Fuller, T.J., Elms, J., Cohen, R. \& White, M.G. (1976) Staphylococcal sepsis in patients on chronic hemodialysis regimens: intravenous treatment with vancomycin given once weekly. Archives of Internal Medicine, 136, 1131.

Boyd, A.D., Spencer, F.C., Isom, O.W., Cunningham, J.N., ReED, G.E., Acinpura, A.J. \& Trice, D.A. (1977) Infective endocarditis. Journal of Thoracic and Cardiovascular Surgery, 73, 23.

Brescia, M.J., Cimino, J.E., Appel, K. \& Hurwich, B.J. (1966) Chronic hemodialysis using venipuncture and a surgically created arteriovenous fistula. New England Journal of Medicine, 275, 1089.

Cross, A.S. \& STeigbigel, R.T. (1976) Infective endocarditis and access site infections in patients on hemodia!ysis. Medicine. Baltimore, 55, 453.

Dathan, J.R.E., Frankel, R.J., Goodwin, F.J., Marsh, J.P., Murray, M., Thompson, J.M.A. \& Youngs, G.R. (1970) One year's experience in a Ministry of Health dialysis centre. British Medical Journal, 2, 102.

Dobbelstein, H. (1976) Immune system in uraemia. Nephron, $17,409$.

Dobkin, J.F., Miller, M.H. \& Sieigbigel, N.H. (1978) Septicemia in patients on chronic hemodialysis. Annals of Internal Medicine, 88, 28.

Dorney, E.R. (1972) Endocarditis. In: The Heart, Arteries and Veins, 2nd edn. (Ed. by Hurst, J.W. \& Logue, R.B.), p. 1168. McGraw-Hill Book Company, New York.

Gombos, E.A., Jefferson, D.M. \& BhAT, J.G. (1975) Effect of haemodialysis on immune response. Proceedings of the European Dialysis and Transplant Association, 11, 367.

Goodman, J.S., Crews, H.D., GinN, H.E. \& Koenig, M.G. (1969) Bacterial endocarditis as a possible complication of chronic hemodialysis. New England Journal of Medicine, 280, 876.

Gregoratos, G. \& Karliner, J.S. (1979) Infective endocarditis: Diagnosis and management. Medical Clinics of North America, 63(1), 173.

Hancock, E.W., Shumway, N.E. \& Remington, J.S. (1971) Valve replacement in active bacterial endocarditis. Journal of Infectious Diseases, 123, 106.

KURTZ, D.M. (1969) Endocarditis after chronic hemodialysis. New England Journal of Medicine, 280, 1302.

Lavelle, K.J. \& Dentino, M.M. (1978) Surgical treatment of infective endocarditis in hemodialysis patients. Clinical Nephrology, 9, 6.

LAWRENCE, H.S. (1965) Uremia-Nature's immunosuppressive device. Annals of Internal Medicine, 62, 166.

Leonard, A., Comty, C.M., Shapiro, F.L. \& RaiJ, L. (1973a) Osteomyelitis in hemodialysis patients. Annals of Internal Medicine, 78, 651.

Lfonard, A., RaiJ, L., Comty, C.M., Wathen, R., ؟ RatTaZzi, T. \& Shapiro, F.L. (1973c) Experience with $\Rightarrow$ endocarditis in a large kidney disease program. Transactions of the American Society of Artificial Internal $\sigma$ Organs, 19, 298.

Leonard, A., RaIJ, L. \& Shapiro, F.L. (1973b) Bacterial endocarditis in regularly dialysed patients. Kidney International, 4, 407.

Lerner, P.I. \& Weinstein, L. (1966) Infective endocarditis in the antibiotic era. New England Journal of Medicine, os 274, 199.

Levi, J., Robson, M. \& Rosenfeld, J.B. (1970) Septicaemia and pulmonary embolism complicating use of arteriovenous fistula in maintenance haemodialysis. Lancet, ii, 288.

McIntosh, C.S., Petrie, J.C. \& Macleod, M. (1969) Maintenance of silastic teflon shunts for intermittent haemodialysis. British Medical Journal, 4, 717.

Martin, A.M., Clunie, G.J.A.. Tonkin, R.W. \& Robson, J.S. (1967) The aetiology and management of shunt infections in patients on intermittent haemodialysis, dialysis and renal transplantation. Proceedings of the European Dialysis and Transplant Association, 4, 67.

Montgomerie, J.Z., Kalmanson, G.M. \& Guze, L.B. (1968) Renal failure and infection. Medicine. Baltimore, 47, 1.

MorRIS, A.J. \& BiLINSKy, R.T. (1971) Prevention of staphylococcal shunt infections by continuous vancomycin $\mathbb{D}$ prophylaxis. American Journal of the Medical Sciences, 262, 87

Newberry, W.M. \& Sanford, J.P. (1971) Defective cellular immunity in renal failure: depression of reactivity of lymphocytes to phytohemagglutinin by renal failurg serum. Journal of Clinical Investigation, 59, 1262.

Nolan, C.M. \& BeATY, H.N. (1976) Staphylococcus aure bacteremia. American Journal of Medicine, 60, 495.

Ralston, A.J., Harlow, G.R., Jones, D.M. \& Davis, $\dot{\mathrm{P}}$ (1971) Infections of Scribner and Brescia arteriovenous shunts. British Medical Journal, 3, 408.

Rao, R., Webster, A.B.D., Sunderland, D.R., Smith, W.F., Ampalan, S. \& LeE, H.A. (1972) Cloxacillin and sodium fusidate in the management of shunt infections. British Medical Journal, 3, 618.

Rebel, M.H., Van Furth, R., Stevens, P., BosscherZonderman, L. \& Noble, W.C. (1975) The flora of renal haemodialysis shunt sites. Journal of Clinical Pathology, 28, 29.

Sherrard, D.J. (1970) Infections in haemodialysis patients. Lancet, ii, 880 .

Wann, L.S., Dillon, J.C., Weyman, A.E. \& Feigenbaum, H. (1976) Echocardiography in bacterial endocarditis. New England Journal of Medicine, 295, 135.

Weinstein, L. \& RuBiN, R.H. (1973) Infective endocarditis1973. Progress in Cardiovascular Diseases, 16, 239.

Weinstein, L. \& Schlessinger, J.J. (1974) Pathoanatomic, pathophysiologic and clinical correlations in endocarditis. New England Journal of Medicine, 291, 832.

Wilson, W.E.C., Kirkpatrick, C.H. \& Talmage, D.W (1965) Suppression of immunologic responsiveness in uremia. Annals of Internal Medicine, 62, 1.

Wing, A.J., Brunner, F.P., Brynger, H., Chantler, C., Donckerwolcke, R.A., Gurland, H.J., Hathaway, R.A. \& JACOBS, C. (1978) Combined report on regular dialysis and transplantation in Europe, VIII, 1977. Proceedings of the European Dialysis and Transplant Association, 15, 4.

WISE, R.I. (1973) Modern management of severe staphylococcal disease. Medicine. Baltimore, 52, 295. 